\title{
Modeling and Analysis of Permanent Magnet Spherical Motors by A Multi-task Gaussian Process Method and Finite Element Method for Output Torque
}

\author{
Yan Wen, Guoli Li, Member, IEEE, Qunjing Wang, Member, IEEE, \\ Xiwen Guo, Member, IEEE, and Wenping Cao, Senior Member, IEEE
}

\begin{abstract}
Permanent magnet spherical motors (PMSMs) operate on the principle of the DC excitation of stator coils and three freedom of motion in the rotor. Each coil generates the torque in a specific direction, collectively they move the rotor to a direction of motion. Modeling and analysis of the output torque are of critical importance for in precise position control applications. The control of these motors requires precise output torques by all coils at a specific rotor position. It is difficult to achieve in the three-dimension space. This paper is the first to apply the Gaussian process to establish the relationship of the rotor position and the output torque for PMSMs. Traditional methods are difficult to resolve such a complex 3D problem with a reasonable computational accuracy and time. This paper utilizes a data-driven method using only input and output data validated by experiments. The multi-task Gaussian process (MTGP) is developed to calculate the total torque produced by multiple coils at the full operational range. The training data and test data are obtained by the finite element method. The effectiveness of the proposed method is validated and compared with existing data-driven approaches. The results exhibit superior performance of accuracy.
\end{abstract}

Manuscript received January 22, 2020; revised May 26, 2020 and July 3, 2020; accepted August 6, 2020. This work was supported in part supported in part by the National Natural Science Foundation of China under Grant 51637001, in part by the Natural Science Research Key Program of Anhui Provincial Education Department under Grant KJ2017A001, and in part by the Young Core Teacher Program of Anhui University under Grant J01005126. (Corresponding authors: Yan Wen; Qunjing Wang.)

Y. Wen is with the School of Computer Science and Technology, Anhui University, Hefei 230601, China, and the School of Electrical Engineering and Automation, Anhui University, Hefei 230601, China, and is also with the National Engineering Laboratory of Energy-Saving Motor \& Control Technology, Anhui University, Hefei 230601, China (e-mail: wenyanchn@gmail.com).

G. Li, X. Guo, and W. Cao are with the School of Electrical Engineering and Automation, Anhui University, Hefei 230601, China (e-mail: liguoli@ahu.edu.cn; xwguo2008@163.com; caowenping@hotmail.com).

Q. Wang is with the Anhui Province Laboratory of Electrical Economize and Safety, Anhui University, Hefei, China (wqunjing@sina.com).
Index Terms-Permanent magnet spherical motor, torque calculation, multi-task Gaussian process.

\section{INTRODUCTION}

SPHERICAL motors are a new type of motor which can $N$ perform multi-degree of freedom (DOF) of motion. In recent years, spherical motor has attracted much attention from academics and practitioners all over the world, as an expected substitute for traditional single-axis motor used in manipulators, satellites, and other multi-DOF devices [1-3]. Similar to traditional electric motors, spherical motors are based on induction motors [4], reluctant motors [5], magnetic levitation motors [6], and permanent magnet motors [7]. Among them, permanent magnet spherical motors (PMSMs) has prevailed owing to their simple structure and compact size.

A wealth of research work has focused on structure optimization [8, 9], attitude detection [10, 11], and position tracking control $[12,13]$. Output torque calculation and modeling of PMSMs of different configurations have been extensively studied as the foundation for positon tracking control. The commonly used method is by means of the finite element method (FEM) based on the virtual displacement method, Maxwell stress tensor method, and Lorentz force method. Its analysis and simplification mainly depend on the structure parameters. [14] designed a multiphase surface-mount PMSM with 112 permanent magnets mounted on the surface of the rotor and 96 electromagnetic coils embedded in the stator. It analyzed the torque characteristics of each coils and derived the output torque equation of the motor by FEM simulations [15, 16]. On one hand, it is difficult to represent by an expression and have to be stored in look-up tables, because the torque characteristic equation are highly nonlinear functions. On the other hand, as the PMs of different layers have different shapes, it is necessary to separately analyze the torque characteristics of every coil on different layers, which greatly increases the computing burden. [17] presented a stepper permanent magnet spherical motor. Its permanent magnets are cylindrical with same size distributed on the rotor evenly, as well as its coils are designed as hollow cylinders with same size and are fixed on the stator uniformly. The clever design of the structure and 
shape of permanents and coils brings an important advantage that the output torque calculation is simplified. The complicated analysis and superposition of total torque characteristics equation is reduced to that of a single coil [18, 19]. This method greatly reduces computational complexity. The torque characteristics of one single coil can be obtained by the polynomial fitting method, instead of being stored in the table. However, this kind of PMSM must be designed in a completely symmetrical structure, which limits the magnetic field distribution and motion continuity. [20] proposed a torque calculation method using a torque map. In the work of [20], a torque generating method for the spherical motor with different permanent magnet arrangements and coil arrangements is discussed. This method has some generality and is a solution of torque nonlinearity caused by the iron-core coils. [21] developed a PMSM based on Halbach array. The air gap magnetic field is closer to a sinusoidal waveform, so that the output torque is larger under the same volume. They used equivalent $2 \mathrm{D}$ model to analyze the torque characteristics of a single coil, which brought errors and also had influence on control precision compared with the 3D model [22, 23].

In general, the mentioned methods of torque calculation depend on the complex electromagnetic analysis and special structure, which are lack of generality for different type of the spherical motors and have some restrictions on motor construction. In order to reduce the complexity of torque calculation, this paper proposes a new torque calculation method based on a Gaussian process for PMSMs. The Gaussian process is a Bayesian modeling approach based on data driven and have widely applied to various machine learning tasks. It is a key merit that Gaussian process is a non-parametric method, which means it allows a model expressivity that naturally calibrated to the requirements of the data [24, 25]. In the field of electrical engineering, Gaussian processes are commonly applied to regression and forecasting, such as short-term solar power forecasting [26], state-of-charge estimations of battery for electric vehicle [27], and power load probability density predictions [28]. In general, Gaussian processes are used to handle with single-output tasks with one or more inputs. However, the torque calculation of a PMSM is a typical multi-output task and the conventional Gaussian processes do not work. To solve this problem, a multi-task Gaussian process is introduced to make an alternative for the torque calculation of the PMSM in this paper. Different from other multi-task models, the multi-task Gaussian process focuses on the correlations between and within tasks and improve the overall accuracy [29].

The major contributions of this work lie in the following three points:

1) It is the first time that the Gaussian process method is utilized for torque calculation of PMSMs. By developing a data-driven method, the complex 3D electromagnetic problem is simplified as a non-parametric regression problem. Once the training set is obtained, the output torque can be calculated by the Gaussian process method without electromagnetic calculation. Moreover, this method can be applied for the spherical motors of different structure in theory, for the calculation of torque is only related to the training set. Therefore, the complexity of torque calculation is reduced significantly without a compromise on accuracy.

2) In order to overcome the problem that the conventional Gaussian process can only generate one output at a time, the proposed multi-task Gaussian process is improved with a multiple output feature, which can generate 24 signals simultaneous for this PMSM.

3) The developed method is compared with existing numerical method (FEM), and two data-driven methods (random forests and k-nearest neighbors) to justify the accuracy and robustness.

\section{StRUCTURE AND MODEL OF A PMSM}

\section{A. Structure of a PMSM}

The overall structure of the PMSM is shown in Fig. 1(a) and the internal structure of the rotor is shown in Fig. 1(b). The PMSM consists of a ball-shaped rotor, a stator composed of two hemispherical shells, and an output shaft fixed on the rotor. There are four layers of 10 equally spaced cylindrical permanent magnets embedded in the rotor and the rotor is supported by several low-friction ball bearings. The $\mathrm{N}$ and $\mathrm{S}$ of permanent magnets in the rotor are arranged in alternation parallel to the equatorial plane. The stator houses two layers of 12 equally spaced electromagnetic coils, through which the currents serve as controlling input to the PMSM. The major specification of the PMSM is shown in Table I.

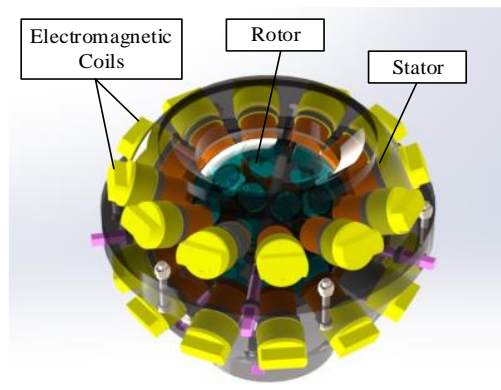

(a)

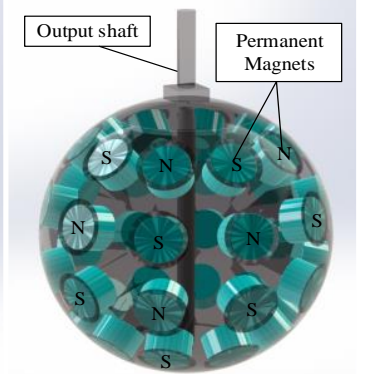

(b)
Fig. 1. Structure of the PMSM: (a) Overall structure of the PMSM; (b) Structure of the rotor with permanent magnets.

TABLE I

SPECIFICATIONS OF THE PMSM

\begin{tabular}{ll}
\hline \hline \multicolumn{1}{c}{ Components } & \multicolumn{1}{c}{ Values } \\
\hline Radius of the stator & $115 \mathrm{~mm}$ \\
Outer radius of coils & $14 \mathrm{~mm}$ \\
Inner radius of coils & $4 \mathrm{~mm}$ \\
Height of coils & $25 \mathrm{~mm}$ \\
Ampere-turns of the coil & $1200 \mathrm{~A}$ \\
Radius of the rotor & $64 \mathrm{~mm}$ \\
Material of permanent magnets & $\mathrm{NdFeB}$ \\
Radius of permanent magnets & $10 \mathrm{~mm}$ \\
Height of permanent magnets & $12 \mathrm{~mm}$ \\
Length of air gap & $1 \mathrm{~mm}$ \\
\hline \hline
\end{tabular}

\section{B. Coordinate Frame and Torque Model}


When a coil is energized by suppling the DC current, a magnetic force is generated by interaction between permanent magnets and electromagnetic field, which propels the rotor to move in a particular direction. Considering that the rotor operates unconstrained in all axes of a rectangular coordinate frame, it is essential to establish a suitable coordinate frame to describe the rotor motion. The rotor coordinate frame $d q p$ and the stator coordinate frame $X Y Z$ (inertia frame) are regulated as shown in Fig. 2. The vector of Euler angles $\boldsymbol{q}=[\alpha, \beta, \gamma]^{\mathrm{T}} \in \mathbb{R}^{3}$ is selected to describe the transformation relationship of two coordinate systems and the homogenous transformation $\boldsymbol{R}_{s r} \in S O(3)$ between is shown in (1).

$$
\boldsymbol{R}_{r s}=\left(\begin{array}{ccc}
\mathrm{c} \beta \mathrm{c} \gamma & \mathrm{s} \alpha \mathrm{s} \beta \mathrm{c} \gamma+\mathrm{s} \alpha \mathrm{c} \gamma & -\mathrm{c} \alpha \mathrm{s} \beta \mathrm{c} \gamma+\mathrm{s} \alpha \mathrm{c} \gamma \\
-\mathrm{c} \beta \mathrm{s} \gamma & -\mathrm{c} \alpha \mathrm{s} \beta c \gamma+\mathrm{s} \alpha \mathrm{c} \gamma & \mathrm{c} \alpha \mathrm{s} \beta \mathrm{s} \gamma+\mathrm{s} \alpha \mathrm{c} \gamma \\
\mathrm{s} \beta & -\mathrm{s} \alpha \mathrm{c} \beta & \mathrm{c} \alpha \mathrm{c} \beta
\end{array}\right)
$$

where $\mathrm{s}$ and $\mathrm{c}$ are the abbreviation of sin and $\cos$, respectively. Thus, the attitude of the rotor can be described by $\boldsymbol{R}_{r s}$.

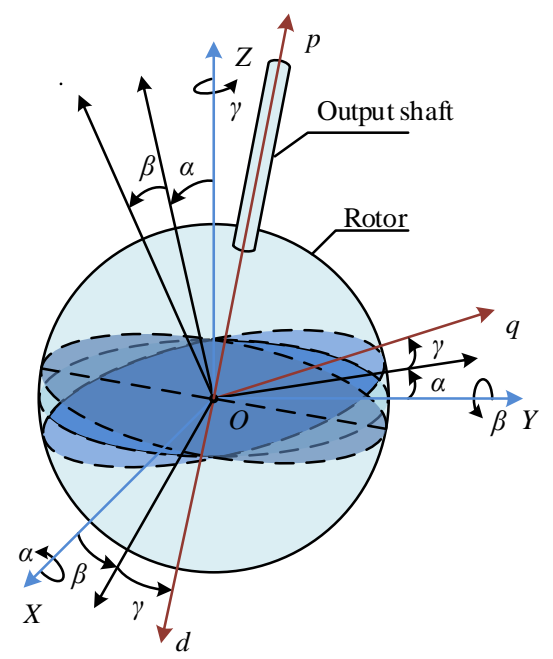

Fig. 2. Coordinate frame.

There is no magnetic iron used in the stator or rotor to transfer magnetic field on their surface. Therefore, the magnetic field saturation effect is ignored. The overall output torque model can be expressed as

$$
\boldsymbol{T}=\left[\begin{array}{c}
T_{x} \\
T_{y} \\
T_{z}
\end{array}\right]=\left[\begin{array}{cccc}
f_{x}^{(1)} & f_{x}^{(2)} & \cdots & f_{x}^{(24)} \\
f_{y}^{(1)} & f_{y}^{(2)} & \cdots & f_{y}^{(24)} \\
f_{z}^{(1)} & f_{z}^{(2)} & \cdots & f_{z}^{(24)}
\end{array}\right] \cdot\left[\begin{array}{c}
i_{1} \\
i_{2} \\
\vdots \\
i_{24}
\end{array}\right]=\boldsymbol{F I}
$$

where $\boldsymbol{T}=\left[T_{x}, T_{y}, T_{z}\right]^{\mathrm{T}} \in \mathbb{R}^{3}$ is the overall output torque with respect to the stator coordinate $X Y Z . I \in \mathbb{R}^{24}$ is the current vector, and $i_{j}$ is the current in the $j$-th coil. $\boldsymbol{F} \in \mathbb{R}^{3 \times 24}$ is the torque matrix by the unit current $(1 \mathrm{~A})$, and $f_{x}^{(j)}, f_{y}^{(j)}$, and $f_{z}^{(j)}$ are the torque contribution coefficients generated by the $j$-th coil around $X-, Y$-, $Z$ - axis, respectively. These coefficients have nonlinear complexity related to the vector of Euler angles $q$ and structure parameters of the PMSM. Considering the vector of Euler angles $q$ as the input, the torque contribution coefficients $f_{x}^{(j)}$ as the output, so as $f_{y}^{(j)}$ and $f_{z}^{(j)}$, the calculation of the torque matrix $\boldsymbol{F}$ can be transformed into several regression problems. Defining $\boldsymbol{f}_{x}, \boldsymbol{f}_{y}, \boldsymbol{f}_{z} \in \mathbb{R}^{24}$, and $\boldsymbol{f}_{x}=\left[f_{x}^{(1)}, f_{x}^{(2)}, \ldots, f_{x}^{(24)}\right]^{\mathrm{T}} \quad, \quad \boldsymbol{f}_{y}=\left[f_{y}^{(1)}, f_{y}^{(2)}, \ldots, f_{y}^{(24)}\right]^{\mathrm{T}} \quad$, $\boldsymbol{f}_{z}=\left[f_{z}^{(1)}, f_{z}^{(2)}, \ldots, f_{z}^{(24)}\right]^{\mathrm{T}}$, the output torque model in (2) can be rewritten as

$$
\boldsymbol{T}=\left[\begin{array}{c}
T_{x} \\
T_{y} \\
T_{z}
\end{array}\right]=\left[\begin{array}{lll}
\boldsymbol{f}_{x} & \boldsymbol{f}_{y} & \boldsymbol{f}_{z}
\end{array}\right]^{\mathrm{T}} \cdot\left[\begin{array}{c}
i_{1} \\
i_{2} \\
\vdots \\
i_{24}
\end{array}\right]=\boldsymbol{F} \boldsymbol{I}
$$

where $\boldsymbol{f}_{x}, \boldsymbol{f}_{y}$, and $\boldsymbol{f}_{z}$ are column vectors of the torque contribution coefficients of all the coils with respect to $X-, Y$, $Z$-axis in the stator coordinate frame. If one considers $\boldsymbol{f}_{x}, \boldsymbol{f}_{y}$, and $\boldsymbol{f}_{z}$ as regression models with multiple outputs related to the Euler angles, the torque matrix $\boldsymbol{F}$ can be described by three regression models. In this paper, the multi-task Gaussian process is utilized to illustrate these models.

\section{MODELING METHOD USING MULTI-TASK GAUSSIAN PROCESS}

The goal of a regression model is to learn the mapping from inputs $x$ to outputs $y$, given a labelled training set of input-output pairs. In this case, define three training set $\mathcal{D}_{x}=\left\{\left(\boldsymbol{x}_{i}, \boldsymbol{y}_{x i}\right) \mid i=1, \ldots, n_{D}\right\} \quad, \quad \mathcal{D}_{y}=\left\{\left(\boldsymbol{x}_{i}, \boldsymbol{y}_{y i}\right) \mid i=1, \ldots, n_{D}\right\} \quad, \quad$ and $\mathcal{D}_{z}=\left\{\left(\boldsymbol{x}_{i}, \boldsymbol{y}_{z i}\right) \mid i=1, \ldots, n_{D}\right\}$, where $\boldsymbol{x}_{i} \in \mathbb{R}^{3}$ denotes the vector of Euler angles $q \cdot \boldsymbol{y}_{x i} \in \mathbb{R}^{24}, \boldsymbol{y}_{y i} \in \mathbb{R}^{24}$, and $\boldsymbol{y}_{z i} \in \mathbb{R}^{24}$ denotes the target vectors of the torque contribution coefficients with respect to $X$-axis, $Y$-axis, and $Z$-axis, respectively.

\section{A. Multi-task Gaussian Process Regression Model under Function-space View}

Because $\mathcal{D}_{x}, \mathcal{D}_{y}$, and $\mathcal{D}_{z}$ are learned in a similar way, the multi-task Gaussian process regression model for $\mathcal{D}_{x}$ is illustrated in detail in this section as an example. When given a training set $\left\{\left(\boldsymbol{x}_{i}, \boldsymbol{y}_{x i}\right) \mid i=1, \ldots, n_{D}\right\}$, it can be assumed that $\boldsymbol{y}_{x i}=\boldsymbol{f}\left(\boldsymbol{x}_{i}\right)$ is a multivariate Gaussian process, which is denoted as

$$
\boldsymbol{f} \sim \mathcal{M G P}\left(\boldsymbol{u}, k_{c}, \Omega\right)
$$

where $\boldsymbol{u}$ is a mean function, $k_{c}$ and $\Omega$ are covariance functions. Assume $\boldsymbol{u}$ is 0 as commonly done in the Gaussian process regression in practice, then, the collection of functions $\left[\boldsymbol{f}\left(\boldsymbol{x}_{1}\right), \boldsymbol{f}\left(\boldsymbol{x}_{2}\right), \ldots, \boldsymbol{f}\left(\boldsymbol{x}_{n_{D}}\right)\right]$ have a joint matrix-variate Gaussian distribution as (5) according to the definition of multivariate Gaussian process (see Appendix A) described in as

$$
\left[\boldsymbol{f}\left(\boldsymbol{x}_{1}\right)^{\mathrm{T}}, \boldsymbol{f}\left(\boldsymbol{x}_{2}\right)^{\mathrm{T}}, \ldots, \boldsymbol{f}\left(\boldsymbol{x}_{n_{D}}\right)^{\mathrm{T}}\right]^{\mathrm{T}} \sim \mathcal{M N}\left(0, \boldsymbol{K}_{c}, \boldsymbol{\Omega}\right)
$$

where $\boldsymbol{K}_{c}$ is a column covariance matrix, of which the $(i, j)$-th element $\left[\boldsymbol{K}_{c}\right]_{i j}=k_{c}\left(\boldsymbol{x}_{i}, \boldsymbol{x}_{j}\right)$, and $\boldsymbol{\Omega}$ is a row covariance matrix.

The joint distribution of the training observations $\boldsymbol{Y}$ at the training locations $X$ and the computed targets $\boldsymbol{f}_{*}$ at the test locations $\boldsymbol{X}_{*}$ are described as 


$$
\left[\begin{array}{c}
\boldsymbol{Y} \\
\boldsymbol{f}_{*}
\end{array}\right] \sim \mathcal{M N}\left(0,\left[\begin{array}{cc}
\boldsymbol{K}_{c}(\boldsymbol{X}, \boldsymbol{X}) & \boldsymbol{K}_{c}\left(\boldsymbol{X}, \boldsymbol{X}_{*}\right) \\
\boldsymbol{K}_{c}\left(\boldsymbol{X}, \boldsymbol{X}_{*}\right)^{\mathrm{T}} & \boldsymbol{K}_{c}\left(\boldsymbol{X}_{*}, \boldsymbol{X}_{*}\right)
\end{array}\right], \boldsymbol{\Omega}\right)
$$

where $\boldsymbol{Y}=\left[\boldsymbol{y}_{1}^{\mathrm{T}}, \boldsymbol{y}_{2}^{\mathrm{T}}, \ldots, \boldsymbol{y}_{n_{D}}^{\mathrm{T}}\right]^{\mathrm{T}}, \quad \boldsymbol{X}=\left[\boldsymbol{x}_{1}, \boldsymbol{x}_{2}, \ldots, \boldsymbol{x}_{n_{D}}\right]^{\mathrm{T}}, \boldsymbol{f}_{*}=\left[\boldsymbol{f}_{* 1}, \boldsymbol{f}_{*_{2}}, \ldots, \boldsymbol{f}_{*_{n_{T}}}\right]^{\mathrm{T}}$, and $\boldsymbol{X}_{*}=\left[\boldsymbol{x}_{*_{1}}, \boldsymbol{x}_{*_{2}}, \ldots, \boldsymbol{x}_{*_{n_{T}}}\right]^{\mathrm{T}}$.

According to the conditional distribution properties of multivariate Gaussian process, the distribution of $\boldsymbol{f}_{*}$ is

$$
p\left(\boldsymbol{f}_{*} \mid \boldsymbol{X}, \boldsymbol{Y}, \boldsymbol{X}_{*}\right) \sim \mathcal{M N}(\hat{\boldsymbol{M}}, \hat{\boldsymbol{\Sigma}}, \hat{\mathbf{\Omega}})
$$

where the mean function matrix $\hat{\boldsymbol{M}}$, and the covariance function matrices $\hat{\boldsymbol{\Sigma}}, \hat{\boldsymbol{\Omega}}$ are given as

$$
\begin{gathered}
\hat{\boldsymbol{M}}=\boldsymbol{K}_{c}\left(\boldsymbol{X}, \boldsymbol{X}_{*}\right)^{\mathrm{T}} \boldsymbol{K}_{c}(\boldsymbol{X}, \boldsymbol{X})^{-1} \boldsymbol{Y} \\
\hat{\boldsymbol{\Sigma}}=\boldsymbol{K}_{c}\left(\boldsymbol{X}_{*}, \boldsymbol{X}_{*}\right)-\boldsymbol{K}_{c}\left(\boldsymbol{X}, \boldsymbol{X}_{*}\right)^{\mathrm{T}} \boldsymbol{K}_{c}(\boldsymbol{X}, \boldsymbol{X})^{-1} \boldsymbol{K}_{c}\left(\boldsymbol{X}, \boldsymbol{X}_{*}\right) \\
\hat{\boldsymbol{\Omega}}=\Omega
\end{gathered}
$$

Furthermore, the expectation and the covariance of $\boldsymbol{f}_{*}$ are derived as

$$
\begin{gathered}
\mathbb{E}\left[\boldsymbol{f}_{*}\right]=\hat{\boldsymbol{M}} \\
\operatorname{cov}\left(\boldsymbol{f}_{*}^{\mathrm{T}}\right)=\hat{\boldsymbol{\Sigma}} \otimes \hat{\mathbf{\Omega}}
\end{gathered}
$$

where $\otimes$ is the Kronecker product.

\section{B. Covariance Matrix}

In the above regression model, the overall covariance matrix is consisted of the column covariance $\hat{\Sigma}$ and the row covariance $\hat{\boldsymbol{\Omega}}$. The covariance $\hat{\boldsymbol{\Sigma}}$ depends on $\boldsymbol{K}_{c}$, which is considered as the kernel matrix associated with training and test inputs.

In order to learn and define the closeness and similarity between data points, the squared exponential (SE) kernel is chosen for its generality and great efficiency in engineering applications [26]. This kernel is infinitely differentiable, which implies that Gaussian process with this kernel have mean square derivatives of all orders, and are thus very smooth [24]. It is suitable for our case because the distribution of torque is theoretically smooth and has no abrupt changes. Considering that the inputs $\boldsymbol{x}_{i}$ is multidimensional, the SE kernel defined by automatic relevance determination (ARD) is adopted. This kernel is commonly called as SEard kernel and is suitable for multidimensional input [24]. The SEard kernel is defined as

$$
k_{c}\left(\boldsymbol{x}_{i}, \boldsymbol{x}_{j}\right)=\sigma_{f}^{2} \exp \left(-\frac{1}{2}\left(\boldsymbol{x}_{i}-\boldsymbol{x}_{j}\right)^{\mathrm{T}} \boldsymbol{P}\left(\boldsymbol{x}_{i}-\boldsymbol{x}_{j}\right)\right)+\delta_{i j} \sigma_{n}^{2}
$$

where $\sigma_{f}^{2}$ represents the variance of the signal; $\delta_{i j}=1$ if $i=j$, otherwise $\delta_{i j}=0 ; \boldsymbol{P}$ is the distance matrix and defined as $\boldsymbol{P}=\operatorname{diag}\left(\ell_{\alpha}, \ell_{\beta}, \ell_{\gamma}\right)^{-2} \cdot \ell_{\alpha}, \ell_{\beta}$, and $\ell_{\gamma}$ are hyper-parameters which play the role of characteristic length-scales in input space. By using SEard kernel, if the length-scale has a very large value, the covariance will become almost independent of that input, effectively removing it from the inference, so that the Gaussian process is capable of feature selection which is of great importance for model learning.

The remaining challenge is to construct the row covariance $\hat{\boldsymbol{\Omega}}$. As $\hat{\boldsymbol{\Omega}}=\Omega$ and $\Omega$ is positive-definite, it can be denoted as $\boldsymbol{\Omega}=\Phi \Phi^{\mathrm{T}}$, where $\Phi$ is a lower triangular matrix such as

$$
\Phi=\left\{\begin{array}{cccc}
\phi_{11} & 0 & \cdots & 0 \\
\phi_{21} & \phi_{22} & \cdots & 0 \\
\vdots & \vdots & \ddots & \vdots \\
\phi_{d 1} & \phi_{d 2} & \cdots & \phi_{d d}
\end{array}\right\}
$$

To guarantee the uniqueness of $\Phi$, the diagonal elements $\phi_{i i}$ for $i=1,2, \ldots, d$ are restricted to be positive. In our case, $d$ is set as the total number of coils. Considering $\psi_{i i}=\ln \left(\phi_{i i}\right)$, the matrix $\Omega$ can be reparameterized by $\left[\psi_{11}, \psi_{22}, \ldots, \psi_{d d}\right]^{\mathrm{T}}$.

\section{Hyper-parameter Optimization}

The free hyper-parameters of the multi-task Gaussian process regression model containing $\ell_{\alpha}, \ell_{\beta}, \ell_{\gamma}, \sigma_{f}^{2}, \sigma_{n}^{2}$ of $\boldsymbol{K}_{c}$ and $\phi_{i j}, \psi_{i i}$ of $\Omega$. These hyper-parameters can be estimated by minimizing the negative log marginal likelihood function from the training data $[30,31]$. According to the matrix-variate distribution, the negative log marginal likelihood function is

$$
\mathcal{L}=\frac{n_{D} d}{2} \ln (2 \pi)+\frac{d}{2} \ln \left|\boldsymbol{K}_{c}\right|+\frac{n_{D}}{2} \ln |\boldsymbol{\Omega}|+\frac{1}{2} \operatorname{tr}\left(\boldsymbol{K}_{c}^{-1} \boldsymbol{Y} \boldsymbol{\Omega}^{-1} \boldsymbol{Y}^{T}\right)
$$

In order to derive derivatives of $\mathcal{L}$ with respect to hyper-parameters, the column covariance matrix $\boldsymbol{K}_{c}$ of the matrix-variate Gaussian distribution in (5) is rewritten as $\boldsymbol{K}_{c}=\boldsymbol{K}_{c 0}+\sigma_{n}^{2} \boldsymbol{E}$, where $\boldsymbol{E}$ is an identity matrix and $\boldsymbol{K}_{c 0}$ is defined as a non-noisy column covariance matrix. The element $\left[\boldsymbol{K}_{c 0}\right]_{i j}$ is the same as the $\left[\boldsymbol{K}_{c}\right]_{i j}$ but without the noisy term $\delta_{i j} \sigma_{n}^{2}$. It should be noted that the hyper-meter set of $\boldsymbol{K}_{c 0}$ is defined as $\Theta=\left\{\theta_{1}, \theta_{2}, \ldots\right\}$. In our case, the elements of the hyper-meter set $\Theta$ are $\sigma_{f}^{2}, \ell_{\alpha}, \ell_{\beta}$, and $\ell_{\gamma}$, which are denoted as $\Theta=\left\{\theta_{i} \mid i=1,2,3,4\right\}$. Then, the hyper-parameters can be classified as $\sigma_{n}^{2}$ of $\boldsymbol{K}_{c}, \theta_{i}$ of $\boldsymbol{K}_{c 0}$, and $\phi_{i j}, \psi_{i i}$ of $\Omega$.

The derivatives of the negative log marginal likelihood function $\mathcal{L}$ in (15) with respect to hyper-parameters $\sigma_{n}^{2}, \theta_{i}$, $\phi_{i j}$, and $\psi_{i i}$ are respectively shown as follows

$$
\begin{gathered}
\frac{\partial \mathcal{L}}{\partial \sigma_{n}^{2}}=\frac{d}{2} \operatorname{tr}\left(\boldsymbol{K}_{c}^{-1}\right)-\frac{1}{2} \operatorname{tr}\left(\boldsymbol{H} \boldsymbol{\Omega}^{-1} \boldsymbol{H}^{\mathrm{T}}\right) \\
\frac{\partial \mathcal{L}}{\partial \theta_{i}}=\frac{d}{2} \operatorname{tr}\left(\boldsymbol{K}_{c}^{-1} \frac{\partial \boldsymbol{K}_{c 0}}{\partial \theta_{i}}\right)-\frac{1}{2} \operatorname{tr}\left(\boldsymbol{H} \boldsymbol{\Omega}^{-1} \boldsymbol{H}^{\mathrm{T}} \frac{\partial \boldsymbol{K}_{c 0}}{\partial \theta_{i}}\right) \\
\frac{\partial \mathcal{L}}{\partial \phi_{i j}}=\frac{n_{D}}{2} \operatorname{tr}\left(\boldsymbol{\Omega}^{-1}\left(\boldsymbol{Q}_{i j} \Phi^{\mathrm{T}}+\Phi \boldsymbol{Q}_{j i}\right)\right) \\
-\frac{1}{2} \operatorname{tr}\left(\boldsymbol{S} \boldsymbol{K}_{c}^{-1} \boldsymbol{S}^{\mathrm{T}}\left(\boldsymbol{Q}_{i j} \Phi^{\mathrm{T}}+\Phi \boldsymbol{Q}_{j i}\right)\right) \\
\frac{\partial \mathcal{L}}{\partial \psi_{i i}}=\frac{n_{D}}{2} \operatorname{tr}\left(\boldsymbol{\Omega}^{-1}\left(\boldsymbol{G}_{i i} \Phi^{\mathrm{T}}+\Phi \boldsymbol{G}_{i i}\right)\right) \\
-\frac{1}{2} \operatorname{tr}\left(\boldsymbol{S} \boldsymbol{K}_{c}^{-1} \boldsymbol{S}^{\mathrm{T}}\left(\boldsymbol{G}_{i i} \Phi^{\mathrm{T}}+\Phi \boldsymbol{G}_{i i}\right)\right)
\end{gathered}
$$

where $\boldsymbol{H}=\boldsymbol{K}_{c}^{-1} \boldsymbol{Y}, \boldsymbol{S}=\boldsymbol{\Omega}^{-1} \boldsymbol{Y}^{T} \cdot \boldsymbol{Q}_{i j}$ and $\boldsymbol{Q}_{j i}$ are square matrices with unities in the $(i, j)$-th and $(j, i)$-th elements, respectively, and zeros elsewhere; $\boldsymbol{G}_{i i}$ is a square matrices with $\exp \left(\psi_{i i}\right)$ in 
the $(i, i)$-th element and zeros elsewhere. In this paper, the Conjugate Gradient method is used to minimize the negative log marginal likelihood function to obtain the estimations of the hyper-parameters.

To sum up, the flow chart of the output torque contribution coefficients calculation process is shown in Fig. 3.

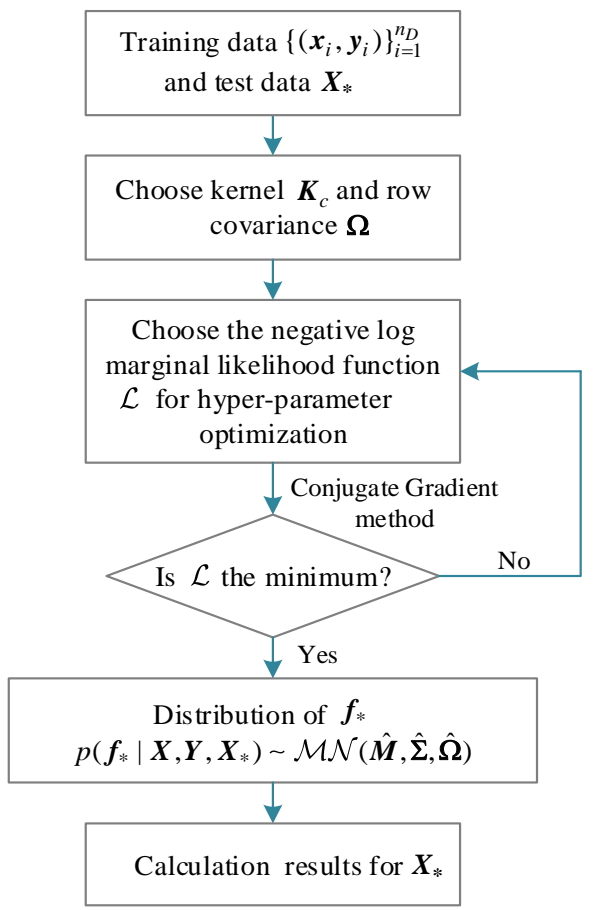

Fig. 3. The flow chart of the output torque contribution coefficients calculation process.

\section{Data Acquisition by Finite Element Method}

The training data are collected by the finite element method. Combining the structural characteristics of the PMSM (see Fig. 1), a 3D finite element model is established in ANSYS and are shown in Fig. 4. The coils are numbered in anticlockwise order to facilitate further description. The analysis is conducted in steady-state. The rotating angles around $X-, Y_{-}$, and $\mathrm{Z}$-axis are assigned to the stator as the variable parameters. They are in correspondence with the Euler angles $\alpha, \beta$, and $\gamma$. Thus, the

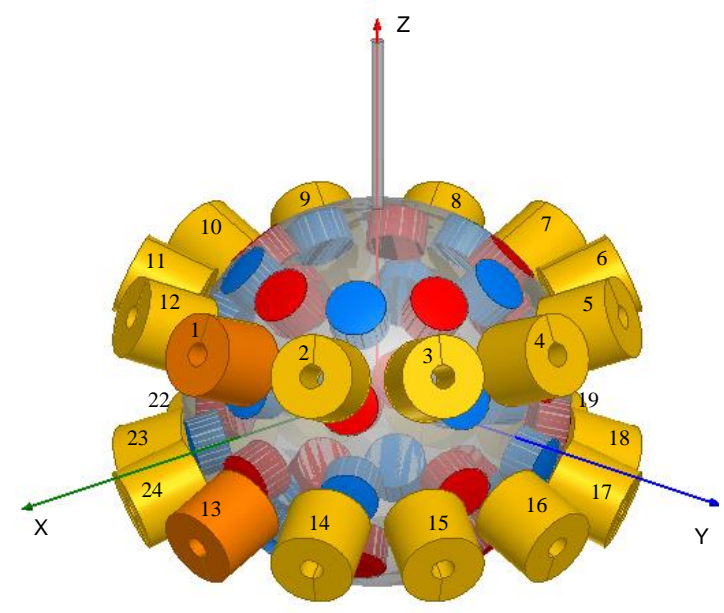

Fig. 4. The 3D model in ANSYS. position of the rotor after motion can be simulated by modifying these parameters. The DC current is added to the cross-section of each coil as an excitation. Then, the output torque around three axes generated by 24 coils at different rotating angles can be analyzed. In order to obtain data efficiently, a simulation method based on Python and ANSYS are used. The diagram of data acquisition and processing is show in Fig. 5. A python-based script sends commands to ANSYS for starting the simulation, modifying the simulation parameters (rotating angles and DC currents), and stopping the simulation. When the script runs, unmanned supervision of simulations can be achieved. This method improves the time-consuming manned method which requires manually modifying parameters and avoids possible manual operation errors.

In this model, the rotating angles corresponding to $\alpha$ and $\beta$ are set in the range of 0 to 37 degrees, and the rotating

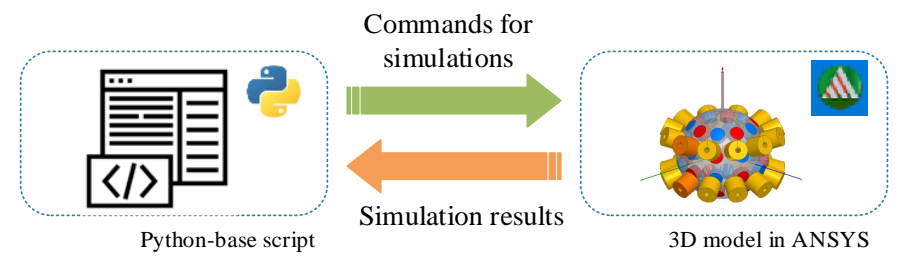

Fig. 5. The diagram of the simulation method based on Python and ANSYS.

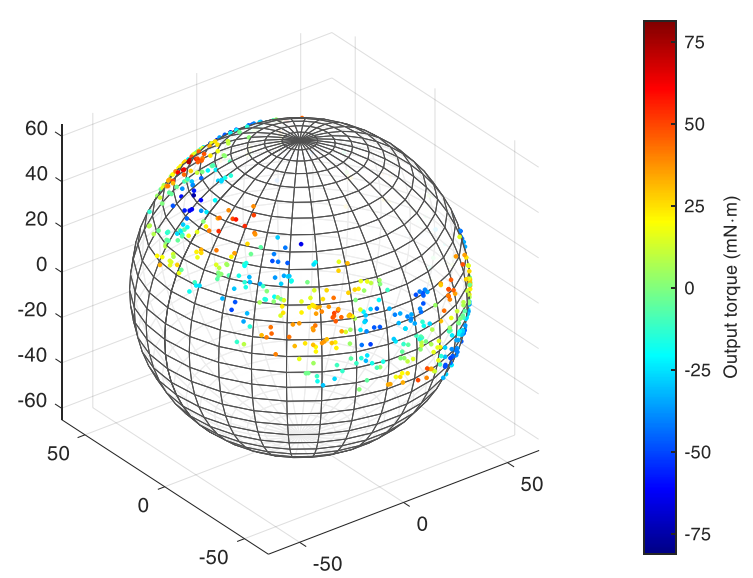

(a)

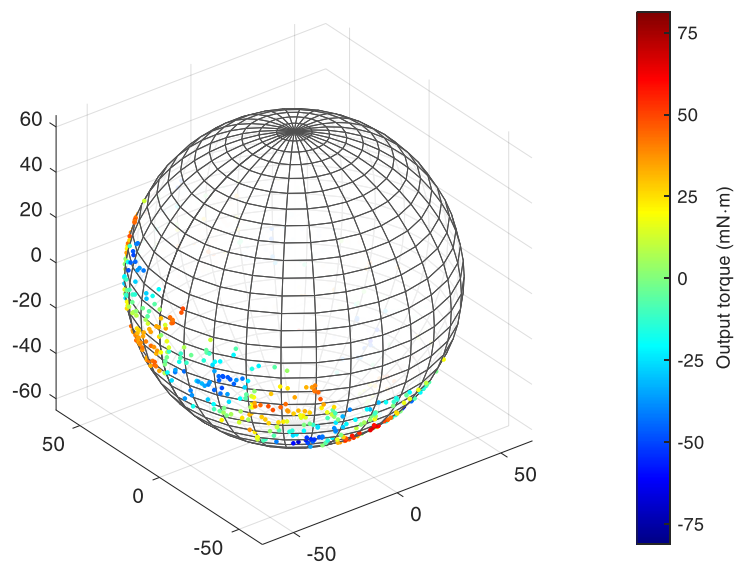

(b)

Fig. 6. The distribution of training data: (a) The 1st coil; (b) The 13th coil. 
angle corresponding to $\gamma$ is set in the range of 0 to 360 degrees. By such the setting, the output shaft of the rotor can move in a maximum range which is limited by the structure of the motor. The dc current through each coil is set as a unit. Then, the torque generated by each coil under the unit current are analyzed by the FEM. For the MTGP learning model, 800 pairs of training date are obtained. Taking the 1 st coil of upper layer and the 13th coil of lower layer for examples, their training data of the output torque around $X$-axis are represented as colored spots distributed on the sphere as shown in Fig. 6. In this figure, the sphere represents the rotor of the PMSM. The position of the colored spot reflects the points on the rotor facing the coil as the rotor rotates at Euler angles and the color of the spot reflects the value of the torque. The color bar on the right illustrates the mapping between colors and values.

\section{EXPERIMENTS}

\section{A. Experimental Setup}

A test bench is established for the validation purpose. It consists of a prototype of the PMSM, a host computer, a current controller, and a torque measurement device mounted on the top of the shaft. The test bench and its hardware implementation are shown in Fig. 7. Due to the 3D motion characteristics of the PMSM, traditional single-axis torque measurement methods using torque transducers are not suitable. Instead, a MEMS gyro sensor is adopted to measure the motion dynamics at starting time, then calculate the output torque with the rotor kinematic equations [32]. Conventionally, the magnetic field may affect the accuracy of the MESM gyro sensor, thus, the MPU-6050 is chosen, which is an integrated sensor combining a 3-axis gyroscope and a 3-axis accelerometer together with an onboard digital motion processor.

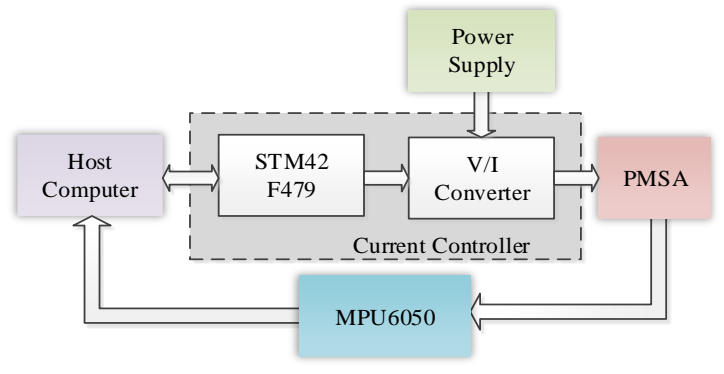

(a)

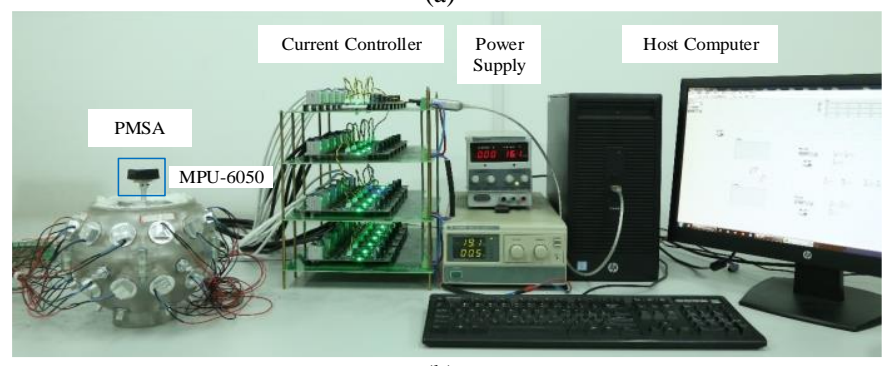

(b)

Fig. 7. The test bench: (a) The diagram; (b) the hardware implementation.

The measuring principle of output torque is briefly illustrated in the followings. When the rotor of the PMSM moves around the constant axis, its dynamics can be expressed according to
Lagrange's Equations of second kind, and its output torque is derived as

$$
T=J \ddot{q}
$$

where $\boldsymbol{J}=\operatorname{diag}\left(J_{x}, J_{y}, J_{z}\right)$ is the inertia matrix and $J_{x}$, $J_{y}, J_{z}$ are the moments of inertia with respect to $X-, Y$-,Z-axis, respectively. $\ddot{\boldsymbol{q}}=[\ddot{\alpha}, \ddot{\beta}, \ddot{\gamma}]^{\mathrm{T}}$ is the vector of Euler angular accelerations. The moments of inertia are obtained by the Automatic Dynamic Analysis of Mechanical Systems (ADAMS). The angular acceleration vector is measured by the MESM gyro sensor. Therefore, the output torque can be calculated by experiments.

\section{B. Validation of FEM Results}

Because the finite element results are utilized to establish the multi-task Gaussian process model, which is then used to compute the output torque, experiments are conducted to verify the feasibility of the finite element results. Considering the output consist of three components around $X-, Y$-, $Z$-axis, the amplitude of the output torque vector $|T|$ is compared in the following verification experiments. Tilt and rotation, which are typical motion cases of the PMSM are considered in the experiments. In the first case, the rotor is initially stationary and will tilt when the 1 st coil and 19th coil are energized by the DC currents with the same magnitude and in the same direction at the same time. Under this condition, the rotor will tilt towards the $X$-axis. The current through each coil is at $0.2 \mathrm{~A}$ intervals from 0.5 to $3 \mathrm{~A}$. In the second case, the rotor is initially stationary and will rotate when the 1 st coil and the 7 th coil are energized by the DC currents with the same magnitude but in opposite direction at the same time. Under this condition, the rotor will rotate around $Z$-axis. The current through each coil is the unit and the output torque is measured at 5-degree intervals from 0 to 180 degrees. In order to compare with the FEM results analyzed in steady-state, the output torque measured at the starting time is adopted. These two motions cases can be analyzed by the FEM when the currents and rotating angles are set. Thus, the analyzed output torque by the FEM can be compared to the experimental results. The results by FEM and experiments are compared as shown in Fig. 8. The red lines

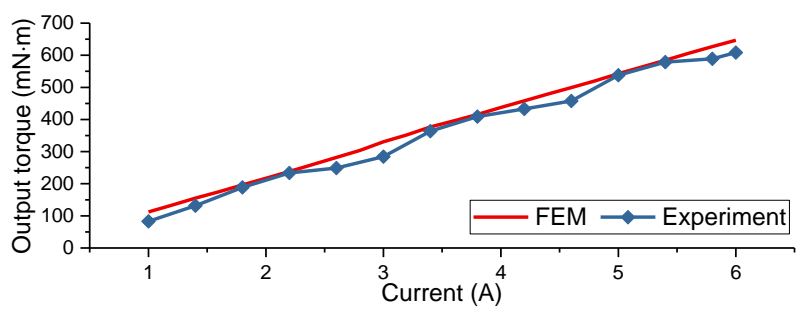

(a)

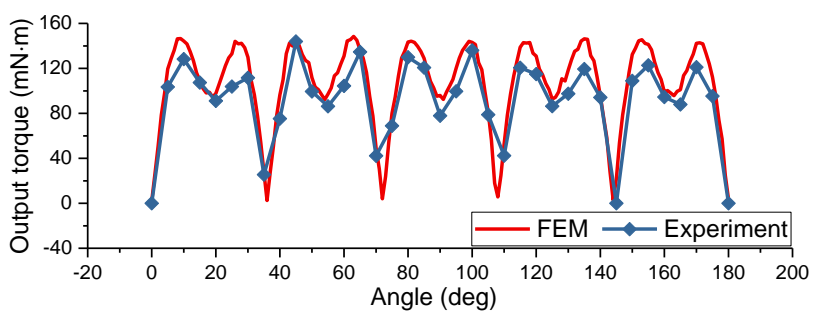

(b)

Fig. 8. The torque by FEM and experiments: (a) The first case; (b) The second case. 
present the output torque analyzed by FEM, and the blue lines present that obtained by experiments. The blue spots present the measurement points. In order to evaluate the comparison on the results by experiments and FEM, the relative error $\Delta$ is defined as

$$
\Delta=\frac{|| \boldsymbol{T}_{e x}|-| \boldsymbol{T}_{\text {fem }} \mid}{\left|\boldsymbol{T}_{\text {fem }}\right|}
$$

where $\boldsymbol{T}_{e x}$ is the measured torque by experiments and $\boldsymbol{T}_{f e m}$ is the analytical torque by FEM. In these figures, there are $1.33 \%-21.88 \%$ relative errors between the experimental and the finite element results. The errors are mainly caused by the implementation of experiments and the friction compensations, which is detailed discussed in [32]. Although the experimental and the finite element results are not perfectly matched with each other, it is clear that the they agree with each other, indicating that that the torque calculated by the finite element method is acceptable. In the following section, the torque analyzed by FEM is used as the training data for the proposed multi-task Gaussian process model.

\section{Validation of the Multi-task Gaussian Process Results}

In the experiments, 800 pairs of the attitude Euler angels and its corresponding output torque around the $X$-axis generated by all the coils energized by a unit current are randomly selected as a training set (see Fig. 6). The proposed multi-task Gaussian
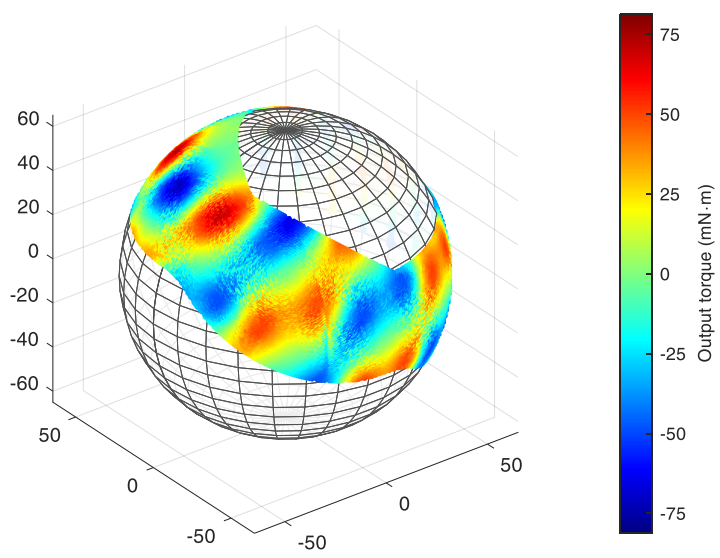

(a)

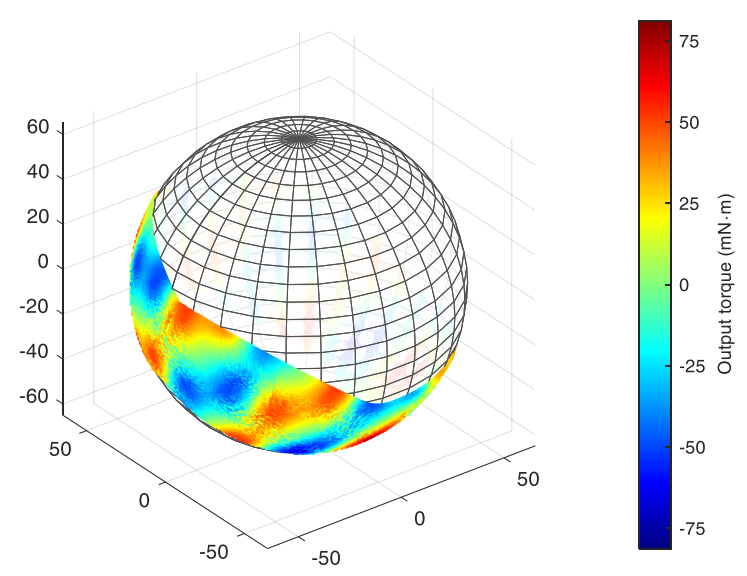

(b)

Fig. 9. The entire distribution of the output torques calculated by the MTGP method: (a) The 1st coil; (b) The 13th coil. process model is used to calculate the overall torque at the full operational range of the output shaft by learning the training data. Taking the 1st coil and the 13th coil as examples, their entire distributions of torque around $X$-axis calculated by the MTGP method are shown in Fig. 9. In order to verify the effectiveness of the MTGP, 50 pairs of the attitude Euler angle and output torque are selected as a test set to compare with the results by the MTGP method. In addition, commonly used data-based learning approaches, random forests (RF) and k-nearest neighbors (KNN), are also used to compared with the proposed method for the 1st coil, the 2nd coil, the 13th coil, and the 14th coil. These results are shown in Fig. 10. The abscissa is the number of the test data, and the output torque in the test set is considered as the reference. It can be found that the torque calculated by the MTGP method matches the reference well. To fully evaluate the performance of these methods, several common performance indicators are adopted and the expressions of these are given in Appendix B. Fig. 11 shows the R-squared score of the MTGP, RF, and KNN method for the 1st coil, the 2 nd coil, the 13th coil, and the 14th coil. It can be seen that the fitting quality for the four coils is the highest by the MTGP method. The lowest R-square score is 0.969 of the proposed method. However, the highest R-square score of the $\mathrm{RF}$ and $\mathrm{KNN}$ method are 0.850 and 0.856 , respectively, which are even lower than the above value.

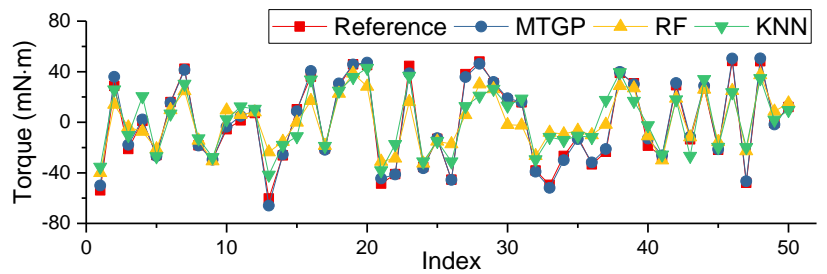

(a)

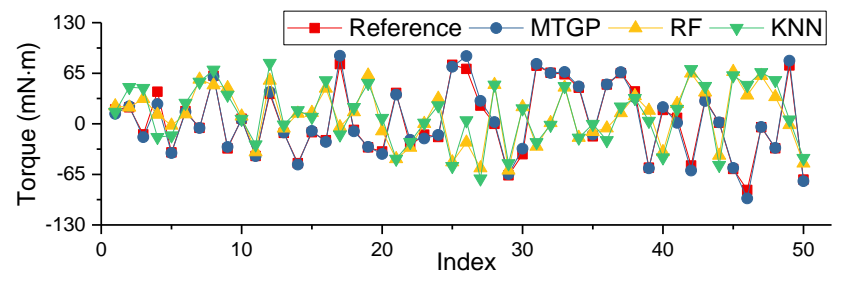

(b)

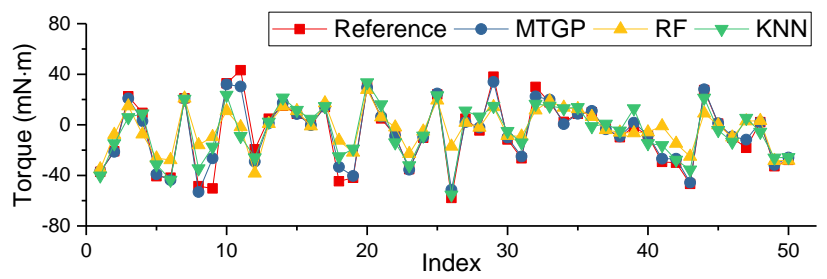

(c)

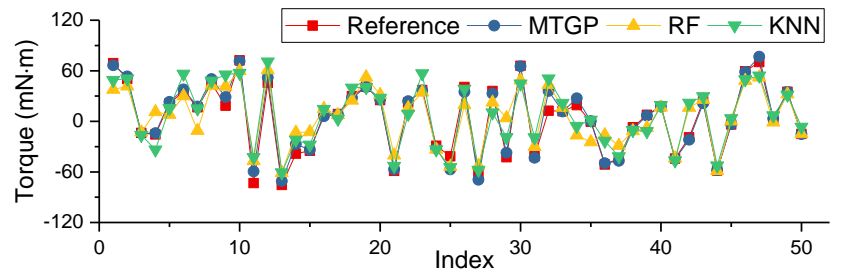

(d)

Fig. 10. Comparisons of MTGP, RF, KNN method: (a) The 1st coil; (b) The 2nd coil; (c) The 13rd coil; (d) The 14th coil. 


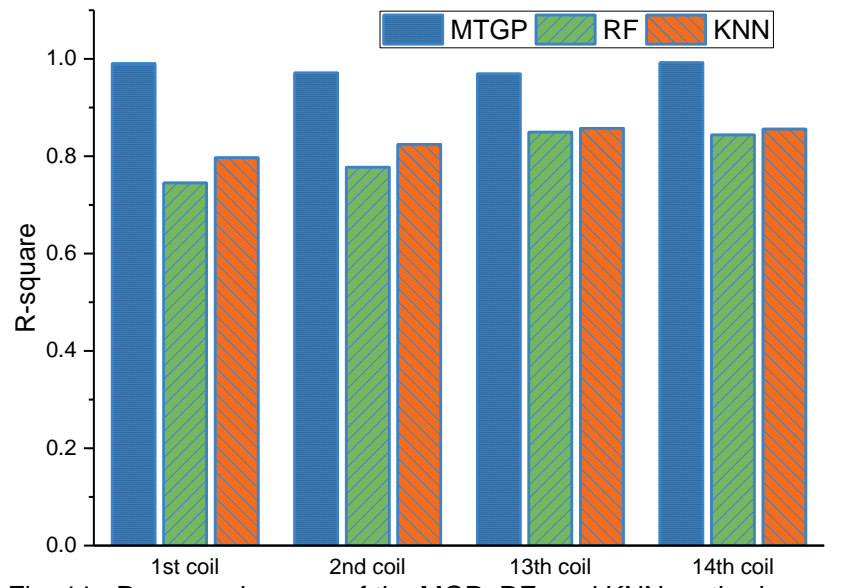

Fig. 11. R-squared scores of the MGP, RF, and KNN methods.

Furthermore, other performance indicators for all the coils, including mean absolute error (MAE), root mean squared error (RMSE), and normalized root mean squared error (nRMSE $\%$ ), are calculated for MTGP, RF and KNN. Fig. 12 shows the distributions of performance indicators for all 24 coils by these methods. The upper and lower bar represent the maximum and the minimum, respectively, and the red line presents the median. It can be seen that the average level of MAEs, RMSEs, and nRMSEs of the MTGP method are lowest compared to other two approaches. The width of the box reflects the volatility level of the indicators. From this figure, the width of the boxes by MTGP are narrower than other two approaches, which indicates better results. The average R-square, MAE, RMSE, $\mathrm{nRMES}_{\%}$ are computed to verify the MTGP, RF, KNN methods as listed in Table II. The running time of the program for the MTGP, RF, KNN methods are listed, too. In terms of the accuracy of the results, it is clear that the MTGP method is superior than the RF and KNN methods. However, in terms of the running time of programs, the MTGP method does not have advantages. With the same size of training set, the MTGP method runs longer than the other two methods. Nevertheless, since the accuracy is of primary importance, the MTGP method is considered to be more appropriate in this case. In fact, the most time-consuming part of this case is obtaining training data

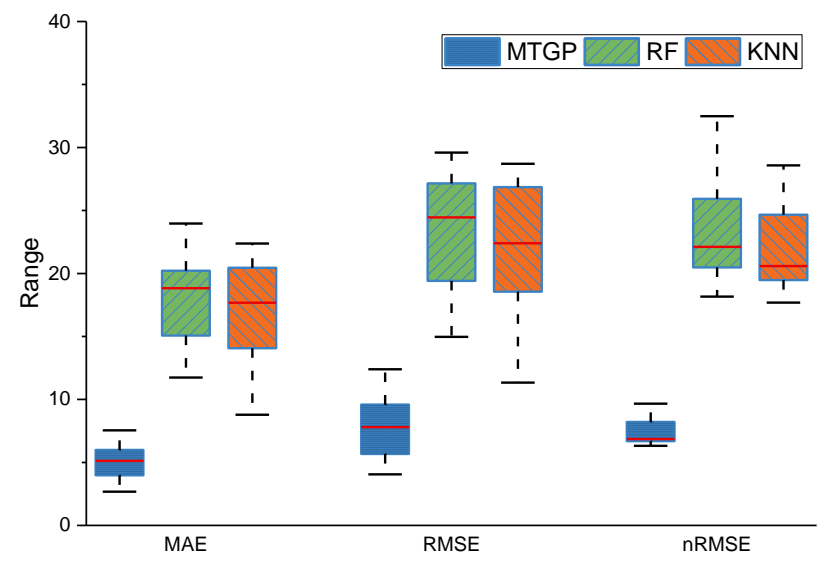

Fig. 12. Distributions of performance indicators for all 24 coils. by the FEM. Therefore, the size of the training set is one of the factors to be taken into account under the same computational accuracy. Table III shows the comparison on the size of the training set among the MTGP, RF, and KNN methods. The average R-square is considered as the performance metric to evaluation these three methods and it is acceptable that the $\mathrm{R}$-squared is greater than 0.95 . From the Table III, it can be found that the size of the training set under the MTGP method is the smallest, indicating that the MTGP method is time-saving in getting training set.

TABLE II

THE AVERAGE PERFORMANCE INDICATORS

\begin{tabular}{lccc}
\hline \hline & MTGP & RF & KNN \\
& & & \\
\hline R-square & 0.980 & 0.805 & 0.832 \\
MAE & 4.952 & 17.949 & 16.833 \\
RMSE & 7.632 & 23.267 & 22.126 \\
nRMSE $_{\%}$ & 7.815 & 23.545 & 22.022 \\
Running time (s) & 28.14 & 9.02 & 7.96 \\
\hline \hline
\end{tabular}

TABLE III

THE SIZE OF TRAINING SET

\begin{tabular}{lccc}
\hline \hline & MTGP & RF & KNN \\
\hline $\begin{array}{l}\text { Size of training } \\
\text { set }\end{array}$ & 600 & 5000 & 4200 \\
R-square & 0.9541 & 0.9503 & 0.9539 \\
\hline \hline
\end{tabular}

\section{CONCLUSION}

This paper has presented a multi-task Gaussian process method to compute the output torques of PMSMs. Test results have validated the numerical method (FEM), which is used to provide training data for the multi-task Gaussian process method. Among data-driven methods, the proposed method has superior performance for PMSMs. The proposed method can be generalized in PMSMs and serves as a guideline for computing output torque using data driven methods. This helps reduce the computation time and promotes the widespread of PMSMs in precise position control applications.

\section{APPENDIX}

\section{A. Matrix-variate Gaussian Distribution}

The random matrix $X \in \mathbb{R}^{n \times d}$ is said to have a matrix-variate Gaussian distribution with mean matrix $\boldsymbol{M} \in \mathbb{R}^{n \times d}$ and covariance matrix $\boldsymbol{\Sigma} \in \mathbb{R}^{n \times n}, \boldsymbol{\Omega} \in \mathbb{R}^{n \times n}$ if and only if its probability density function is given by

$$
\begin{gathered}
p(\boldsymbol{X} \mid \boldsymbol{M}, \boldsymbol{\Sigma}, \boldsymbol{\Omega})=(2 \pi)^{-d n / 2} \operatorname{det}(\boldsymbol{\Sigma})^{-d / 2} \operatorname{det}(\boldsymbol{\Omega})^{-n / 2} \\
\times \operatorname{etr}\left(-\frac{1}{2} \boldsymbol{\Omega}^{-1}(\boldsymbol{X}-\boldsymbol{M})^{\mathrm{T}} \boldsymbol{\Sigma}^{-1}(\boldsymbol{X}-\boldsymbol{M})\right)
\end{gathered}
$$

where $\operatorname{etr}(\cdot)$ is exponential of matrix trace, $\boldsymbol{\Omega}$ and $\boldsymbol{\Sigma}$ are positive semi-definite. It is denoted $\boldsymbol{X} \sim \mathcal{M \mathcal { N } _ { d , n }}(\boldsymbol{M}, \boldsymbol{\Sigma}, \boldsymbol{\Omega})$.

\section{B. Performance Indicators}


In Table IV, $y_{r, i}$ refers to the $i$-th reference value, and $y_{p, i}$ refers to the $i$-th predictive value.

TABLE IV

PERFORMANCE INDICATORS AND THEIR EXPRESSIONS

\begin{tabular}{|c|c|}
\hline Performance indicator & Expression \\
\hline R-squared & $R^{2}=1-\frac{\sum_{i=1}^{N}\left(y_{r, i}-y_{p, i}\right)^{2}}{\sum_{i=1}^{N}\left(y_{r, i}-\frac{1}{N} \sum_{i=1}^{N} y_{r, i}\right)^{2}}$ \\
\hline Mean Absolute Error & $M A E=\frac{1}{N} \sum_{i=1}^{N}\left|y_{r, i}-y_{p, i}\right|$ \\
\hline $\begin{array}{l}\text { Root Mean Squared } \\
\text { Error }\end{array}$ & $R M S E=\sqrt{\frac{1}{N} \sum_{i=1}^{N}\left(y_{r, i}-y_{p, i}\right)^{2}}$ \\
\hline $\begin{array}{l}\text { Normalized Root Mean } \\
\text { Squared Error }\end{array}$ & $n R M S E_{\%}=\frac{\sqrt{\frac{1}{N} \sum_{i=1}^{N}\left(y_{r, i}-y_{p, i}\right)^{2}}}{\max \left\{y_{r, i}\right\}}$ \\
\hline
\end{tabular}

\section{REFERENCES}

[1] D. K. Kim, H. Yoon, W. Y. Kang, Y. B. Kim, and H. T. Choi, "Development of a spherical reaction wheel actuator using electromagnetic induction," Aerospace Science and Technology, vol. 39, pp. 86-94, Dec 2014.

[2] H. Y. Kim, H. Kim, D. G. Gweon, and J. Jeong, "Development of a novel spherical actuator with two degrees of freedom," IEEE-ASME Transactions on Mechatronics, Article vol. 20, no. 2, pp. 532-540, Apr 2015.

[3] K. Bai, K. M. Lee, J. Cao, R. Y. Xu, and L. Li, "Design and decoupled compensation methods of a pm motor capable of 6-d force/torque actuation for minimum bearing reaction," IEEE-ASME Transactions on Mechatronics, vol. 22, no. 5, pp. 2252-2264, Oct 2017.

[4] J. F. P. Fernandes and P. J. C. Branco, "The shell-like spherical induction motor for low-speed traction: electromagnetic design, analysis, and experimental tests," IEEE Transactions on Industrial Electronics, vol. 63, no. 7, pp. 4325-4335, Jul 2016.

[5] Y. B. Kim, J. S. Lee, and B. I. Kwon, "Experiment and torque modeling of double-excited, two-degree-of-freedom motor based on magnetic equivalent circuit analysis," Journal of Electrical Engineering \& Technology, Article vol. 8, no. 1, pp. 130-136, Jan 2013.

[6] Z. Li and Q. J. Wang, "Levitation mechanism and improvements of 3-dof deflection type pm actuator," IEEE Transactions on Applied Superconductivity, vol. 26, no. 7, pp. 1-5, Oct 2016.

[7] J. Wang, G. W. Jewell, and D. Howe, "Analysis, design and control of a novel spherical permanent-magnet actuator," IEE Proceedings - Electric Power Applications, vol. 145, no. 1, pp. 61-71, 1998.

[8] L. Yan, I. M. Chen, C. K. Lim, G. L. Yang, and K. M. Lee, "Modeling and iron-effect analysis on magnetic field and torque output of electromagnetic spherical actuators with iron stator," IEEE-ASME Transactions on Mechatronics, vol. 17, no. 6, pp. 1080-1087, Dec 2012.

[9] L. Yan, F. Liang, Z. Jiao, and T. Wang, "Magnetic field analysis of novel spherical actuators with three-dimensional pole arrays," Rev Sci Instrum, vol. 87, no. 6, p. 065006, Jun 2016, Art. no. 065006.

[10] Y. Lu, C. G. Hu, Q. J. Wang, Y. Hong, W. X. Shen, and C. Q. Zhou, "A new rotor position measurement method for permanent magnet spherical motors," Applied Sciences-Basel, vol. 8, no. 12, Dec 2018.

[11] Y. P. Rong, Q. J. Wang, S. L. Lu, G. L. Li, Y. Lu, and J. Z. Xu, "Improving attitude detection performance for spherical motors using a MEMS inertial measurement sensor," IET Electric Power Applications, vol. 13, no. 2, pp. 198-205, Feb 2019.

[12] J. M. Liu, H. Y. Deng, W. H. Chen, and S. P. Bai, "Robust dynamic decoupling control for permanent magnet spherical actuators based on extended state observer," IET Control Theory and Applications, vol. 11, no. 5, pp. 619-631, Mar 172017.
[13] Y. Wen, G. Li, Q. Wang, and X. Guo, "Robust adaptive sliding-mode control for permanent magnet spherical actuator with uncertainty using dynamic surface approach," Journal of Electrical Engineering \& Technology, vol. 14, no. 6, pp. 2341-2353, 2019.

[14] K. Kahlen and R. W. De Doncker, "Current regulators for multi-phase permanent magnet spherical machines," presented at the IEEE Industry Applications Conference, Rome, Italy, 8-12 Oct, 2000.

[15] K. Kahlen and R. W. De Doncker, "Control of multi-dimensional drives with variable pole pitch," presented at the IEEE Industry Applications Conference, Pittsburgh, PA, USA, 13-18 Oct., 2002.

[16] K. Kahlen, I. Voss, C. Priebe, and R. W. De Doncker, "Torque control of a spherical machine with variable pole pitch," IEEE Transactions on Power Electronics, vol. 19, no. 6, pp. 1628-1634, Nov 2004.

[17] K. M. Lee and H. Son, "Torque model for design and control of a spherical wheel motor," in IEEE/ASME International Conference on Advanced Intelligent Mechatronics. Proceedings, 2005, pp. 335-340.

[18] H. Son and K. M. Lee, "Distributed multipole models for design and control of PM actuators and sensors," IEEE-ASME Transactions on Mechatronics, vol. 13, no. 2, pp. 228-238, Apr 2008.

[19] K. M. Lee, K. Bai, and J. Lim, "Dipole models for forward/inverse torque computation of a spherical motor," IEEE-ASME Transactions on Mechatronics, vol. 14, no. 1, pp. 46-54, Feb 2009.

[20] N. Kasashima, K. Ashida, T. Yano, A. Gofuku, M. Shibata, "Torque control method of an electromagnetic spherical motor using torque map," IEEE-ASME Transactions on Mechatronics, vol. 21, No. 4, pp.2050-2060, 2016.

[21] C. L. Xia, H. F. Li, and T. N. Shi, "3-D magnetic field and torque analysis of a novel halbach array permanent-magnet spherical motor," IEEE Transactions on Magnetics, vol. 44, no. 8, pp. 2016-2020, Aug 2008.

[22] X. Changliang, S. Peng, L. Hongfeng, L. Bin, and S. Tingna, "Research on torque calculation method of permanent-magnet spherical motor based on the finite-element method," IEEE Transactions on Magnetics, vol. 45, no. 4, pp. 2015-2022, 2009

[23] B. Li, G. D. Li, and H. F. Li, "Magnetic field analysis of 3-dof permanent magnetic spherical motor using magnetic equivalent circuit method," IEEE Transactions on Magnetics, vol. 47, no. 8, pp. 2127-2133, Aug 2011.

[24] C. E. Rasmussen and C. K. I. Williams, Gaussian Process for Machine Learning. London, England: The MIT Press, 2006.

[25] N. D. Lawrence, L. Rosasco, and M. A. Álvarez, "Kernels for vector-valued functions: a review," Foundations and Trends in Machine Learning, vol. 4, no. 3, pp. 195-266, 2012.

[26] H. M. Sheng, J. Xiao, Y. H. Cheng, Q. Ni, and S. Wang, "Short-Term Solar Power Forecasting Based on Weighted Gaussian Process Regression," IEEE Transactions on Industrial Electronics, vol. 65, no. 1, pp. 300-308, Jan 2018.

[27] H. M. Sheng, J. Xiao, and P. Wang, "Lithium iron phosphate battery electric vehicle state-of-charge estimation based on evolutionary gaussian mixture regression," IEEE Transactions on Industrial Electronics, vol. 64, no. 1, pp. 544-551, Jan 2017.

[28] Y. D. Yang, S. F. Li, W. Q. Li, and M. J. Qu, "Power load probability density forecasting using Gaussian process quantile regression," Applied Energy, vol. 213, pp. 499-509, Mar 12018.

[29] F. Rodrigues, K. Henrickson, and F. C. Pereira, "Multi-output gaussian processes for crowdsourced traffic data imputation," IEEE Transactions on Intelligent Transportation Systems, vol. 20, no. 2, pp. 594-603, Feb 2019.

[30] B. Wang, and T. Chen, "Gaussian process regression with multiple response variables," Chemometric and Intellighent Laboratory Systems, vol. 142, no. 15, pp. 159-165, Mar 2015.

[31] Z. Chen, B. Wang and A. N. Gorban, "Multivariate gaussian and student $-\mathrm{t}$ process regression for multi-output prediction," Neural Computing and Applications, vol. 32, pp. 3005-3028, Apr 2020.

[32] Y. Wen, G. Li, Q. Wang, R. Tang, Y. Liu, and H. Li, "Investigation on the measurement method for output torque of a spherical motor," Applied Sciences-Basel, vol. 10, no. 7, pp. 2510, Apr 2020.

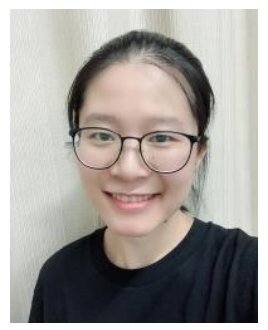

Yan Wen was born in Hefei, China, in 1990. She received the B.Eng. degree in electrical engineering from Qiqihar University, Qiqihar, China, in 2012. She is currently a Ph.D. candidate in computer science and technology at Anhui University, Hefei, China. 
Her research interests include intelligent control theory and spherical motors, particularly modelling, attitude detection, and intelligent control method of spherical motors.

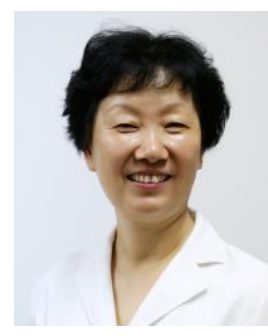

Guoli Li received the B.Eng. degree in electrical engineering from Hefei University of Technology, Hefei, China, in 1983, and the Ph.D. degree in nuclear science and engineering from Hefei Institutes of Physical Science, Chinese Academy of Sciences, Hefei, China, in 2006.

She is currently a Professor with the School of Electrical Engineering and Automation, Anhui University, Hefei, China. She is involved in teaching and research of electric motors and electric drives.

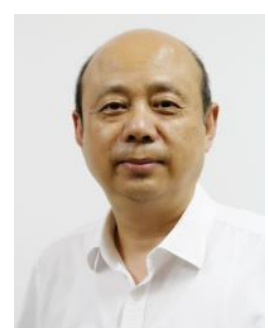

Qunjing Wang received the B.Eng. degree in electrical engineering from Hefei University of Technology, Hefei, China, in 1982, and the Ph. D degree from University of Science and Technology of China, Hefei, China, in 1998.

$\mathrm{He}$ is currently a Professor with Anhui University, Hefei, China, and a director with the National Engineering Laboratory of Energy-Saving Motor \& Control Technology, Anhui University, Hefei, China. He is also in a head position with the Anhui Province Laboratory of Electrical Economize and Safety, Anhui University, Hefei, China. He is involved in research of electric motors, electric drives, and power electronics.

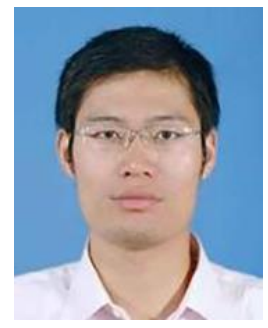

Xiwen Guo received the B.Eng. degree in electrical engineering from Anhui University of Science and Technology, Huainan, China, in 2005, and the Ph.D. degree in electrical engineering from Hefei University of Technology, Hefei, China, in 2012.

$\mathrm{He}$ is currently an Associate Professor with the School of Electrical Engineering and Automation, Anhui University, Hefei, China. His research interests include special motor and its control, power electronics and electric drives, intelligent control, and motor-pump modeling.

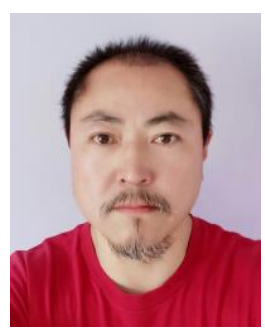

Wenping Cao (M'05-SM'11) received the B.Eng in electrical engineering from Beijing Jiaotong University, Beijing, China, in 1991, and the Ph.D. degree in electrical machines and drives from the University of Nottingham, Nottingham, U.K., in 2004.

$\mathrm{He}$ is currently a Chair Professor of Electrical Power Engineering at Aston University, Birmingham, U.K., and also a Visiting Professor at School of Electrical Engineering and Automation, Anhui University, P. R. China. His research interests include fault analysis and condition monitoring of electrical machines and power electronics.

Prof. Cao is the Chairman for the Industrial Electronics Society, IEEE UK and Ireland Section, and also a "Royal Society Wolfson Research Merit Award" holder, U.K. He was a semi-finalist at the "Annual MIT-CHIEF Business Plan Contest", U.S.A., in 2015; the "Dragon's Den Competition Award" winner from Queen's University Belfast, U.K., in 2014, the "Innovator of the Year Award" winner from Newcastle University, U.K., in 2013. He received the "Best Paper Awards" from the IET International Conference on Renewable Power Generation (RPG) in 2019, and the 9th International Symposium on Linear Drives for Industry Applications (LDIA) in 2013. 